\title{
The Protective Effect of Melissa officinalis L. in Visceral Hypersensitivity in Rat Using 2 Models of Acid-induced Colitis and Stress-induced Irritable Bowel Syndrome: A Possible Role of Nitric Oxide Pathway
}

\begin{abstract}
Fatemeh Dolatabadi, ${ }^{1}$ Amir H Abdolghaffari, ${ }^{2,3,4}$ Mohammad H Farzaei, $^{5,6}$ Maryam Baeeri, $^{3}$ Fatemeh S Ziarani, $^{7}$ Majid Eslami, ${ }^{3}$ Mohammad Abdollahi, ${ }^{3}$ and Roja Rahimi ${ }^{8 *}$

${ }^{1}$ Faculty of Pharmacy, Tehran University of Medical Sciences, Tehran, Iran; ${ }^{2}$ Medicinal Plants Research Center, Institute of Medicinal Plants, ACECR, Karaj, Iran; ${ }^{3}$ Department of Pharmacology and Toxicology and Pharmaceutical Sciences Research Center, Faculty of Pharmacy, Tehran University of Medical Sciences, Tehran, Iran; ${ }^{4}$ Gastrointestinal Pharmacology Interest Group (GPIG), Universal Scientific Education and Research Network (USERN), Tehran, Iran; ${ }^{5}$ Pharmaceutical Sciences Research Center and ${ }^{6}$ Medical Biology Research Center, Kermanshah University of Medical Sciences, Kermanshah, Iran; ${ }^{7}$ Department of Anatomy, School of Medicine, Ghazvin University of Medical Sciences, Ghazvin, Iran; and ${ }^{8}$ Department of Traditional Pharmacy, School of Traditional Medicine, Tehran University of Medical Sciences, Tehran, Iran
\end{abstract}

\section{Background/Aims}

The aim of present study is to estimate the effects of Melissa officinalis L. (MO) on visceral hypersensitivity (VH), defecation pattern and biochemical factors in 2 experimental models of irritable bowel syndrome (IBS) and the possible role of nitric oxide.

\section{Methods}

Two individual models of IBS were induced in male Wistar-albino rats. In the acetic acid model, the animals were exposed to rectal distension and abdominal withdrawal reflex, and the defecation patterns were determined. In the restraint stress model, the levels of TNF- $\alpha$, myeloperoxidase, lipid peroxidation, and antioxidant powers were determined in the (removed) colon. Rats had been treated with $\mathrm{MO}$, L-NG-nitroarginine methyl ester (L-NAME), aminoguanidine (AG), MO + AG, or MO + L-NAME in the mentioned experimental models.

\section{Results}

Hypersensitive response to rectal distension and more stool defecation in control rats have been observed in comparison to shams. MO-300 significantly reduced VH and defecation frequency in comparison to controls. VH and defecation pattern did not show significant change in AG + MO and L-NAME + MO groups compared to controls. Also, significant reduction in TNF- $\alpha$, myeloperoxidase, thiobarbituric acid reactive substances (TBARS), and an increase in antioxidant power in MO-300 group was recorded compared to controls. AG + MO and L-NAME + MO groups showed a reverse pattern compared to MO-300 group.

\section{Conclusions}

MO can ameliorate IBS by modulating VH and defecation patterns. Antioxidant and anti-inflammatory properties along with its effect on the nitrergic pathway seem to play important roles in its pharmacological activity.

(J Neurogastroenterol Motil 2018;24:490-501)

Key Words

Irritable bowel syndrome; Lemon balm; Melissa officinalis; Nitric oxide; Visceral hypersensitivity

Received: March 6, 2017 Revised: November 25, 2017 Accepted: January 21, 2018

(a) This is an Open Access article distributed under the terms of the Creative Commons Attribution Non-Commercial License (http://creativecommons. org/licenses/by-nc/4.0) which permits unrestricted non-commercial use, distribution, and reproduction in any medium, provided the original work is properly cited.

${ }^{*}$ Correspondence: Roja Rahimi, MD Department of Traditional Pharmacy, School of Traditional Medicine, Tehran University of Medical Sciences, Tehran 1416663547, Iran Tel: +98-21-88990835, Fax: +98-21-88990835, E-mail: rojarahimi@gmail.com

Fatemeh Dolatabadi and Amir H Abdolghaffari contributed equally in this work. 


\section{Introduction}

Irritable bowel syndrome (IBS) is the most prevalent ailment referred to gastroenterologists with a reported overall prevalence of approximately $11.2 \%{ }^{1}$ IBS is generally characterized by symptoms such as abdominal pain and discomfort, bloating, altered bowel habit, and disturbed gastrointestinal motility, among which abdominal pain have been seen in almost all the patients. ${ }^{2}$ These symptoms are associated with miscommunication between the neural, endocrine and immune systems. The pathophysiology of IBS is not fully understood; however, gastrointestinal infections, psychosocial stressors, as well as low-grade inflammation in the gastrointestinal tract due to the activation of mucosal immune system can be considered as the main contributing factors in the pathophysiology of the disease. ${ }^{3}$ Increase in myeloperoxidase (MPO) activity, TNF- $\alpha$, IL-1 $\beta$, IL-6, and more activated neutrophil and eosinophil in patients with IBS compared to healthy subjects confirmed the role of inflammatory reaction in the pathogenesis. ${ }^{4,5}$

Visceral hypersensitivity $(\mathrm{VH})$ is proposed as one of the major factors involved in the pathophysiology of IBS. VH is a manifestation of biopsychosocial disorders such as functional dyspepsia or IBS. ${ }^{6}$ It leads to changes in gut motility by troubling regulatory reflex pathways and secretory function. Some reports have suggested the involvement of nitric oxide (NO), a free radical that is produced from L-arginine by $\mathrm{NO}$ synthases (NOSs), in pain transmission. ${ }^{7,8}$

Despite various categories of conventional drugs including antispasmodics, antidepressants, antibiotics, and probiotics used for the management of IBS, the results have not been encouraging. ${ }^{9}$ Thus, seeking alternative agents for management of this disease seems important. Medicinal plants are proposed as a valuable source for discovering and identifying new pharmacological agents. ${ }^{9-11}$

Melissa officinalis L. (MO), a perennial herbaceous plant from the family Lamiaceae and commonly known as lemon balm, grows worldwide; however, the Eastern Mediterranean region, Western Asia and Southern Europe, Caucasus, and Northern Iran are considered as its origin. $\mathrm{MO}$ has been traditionally used for different medicinal purposes including CNS disorders such as nervous agitation; sleep disturbances, depression, as well as gastrointestinal problems such as indigestion associated with nervous tension and flatulence. ${ }^{12-14}$ Previous studies have demonstrated spasmolytic, ${ }^{15}$ immunostimulant, ${ }^{16}$ anti-inflammatory, ${ }^{17}$ antioxidant activity ${ }^{18}$ anxiolytic, antidepressant, and anti-stress activities of $\mathrm{MO}^{19-21}$ The major chemical constituents of $\mathrm{MO}$ are essential oil components (mostly composed of geranial and neral), hydroxycinnamic acid de- rivatives, flavonoids, and tannins. ${ }^{15,22}$

Taking into consideration the biological activities of $\mathrm{MO}$ on both the nervous system and gastrointestinal tract, we decided to investigate the effect of hydroethanolic extract of $\mathrm{MO}$ on $\mathrm{VH}$ and other symptoms of IBS, and the possible role of IBS-associated inflammatory reactions as well as the nitrergic pathway in its therapeutic properties in 2 different experimental models of IBS.

\section{Materials and Methods}

\section{Chemicals}

Trichloroacetic acid (TCA), thiobarbituric acid (TBA), 2,4,6-Tri(2-pyridyl)-s-triazine (TPTZ), n-butanol, hexadecyl trimethyl ammonium bromide (HETAB), ethylene diamine tetra acetic acid (EDTA), malondialdehyde (MDA), hydrochloric acid $(\mathrm{HCl})$, acetic acid, hydrogen peroxide $\left(\mathrm{H}_{2} \mathrm{O}_{2}\right)$, O-dianisidine hydrochloride, ferric chloride $\left(\mathrm{FeCl}_{3}-6 \mathrm{H}_{2} \mathrm{O}\right)$, Coomassie reagent, bovine serum albumin, sodium sulfate $\left(\mathrm{Na}_{2} \mathrm{SO}_{4}\right)$, sulfuric acid $\left(\mathrm{H}_{2} \mathrm{SO}_{4}\right)$, potassium dihydrogen phosphate $\left(\mathrm{KH}_{2} \mathrm{PO}_{4}\right)$, potassium hydrogen diphosphate $\left(\mathrm{K}_{2} \mathrm{HPO}_{4}\right)$, sodium carbonate $\left(\mathrm{Na}_{2} \mathrm{CO}_{3}\right)$, n-butanol, phosphate buffer, and ethanol from Merck (Darmstadt, Germany), rat-specific TNF- $\alpha$ and interleukin- $1 \beta$ ELISA kits (from Bender MedSystems GmbH, Austria), trichloroacetic acid $\left(\mathrm{CCl}_{3} \mathrm{COOH}\right)$, ether, tetraethoxypropane, L-NG-Nitroarginine Methyl Ester (L-NAME), aminoguanidine (AG), and acetic acid (Fluka, Buchs, Switzerland) were used in this study.

\section{Plant Material}

Dried aerial parts of $\mathrm{MO}$ were purchased from the local herbal market of Tehran (Iran). The plant was confirmed and a voucher specimen (PMP-407) was deposited in the Herbarium of the Department of Pharmacognosy, Faculty of Pharmacy, Tehran University of Medical Sciences, Tehran, Iran.

\section{Extract Preparation}

For preparation of hydroalcoholic extract of MO, $200 \mathrm{~g}$ of dried aerial parts of $\mathrm{MO}$ were powdered and $200 \mathrm{~g}$ of the powder was extracted by maceration at room temperature $\left(24 \pm 3^{\circ} \mathrm{C}\right)$ using $600 \mathrm{~mL}$ of ethanol (70\%) as solvent for 72 hours. The extract was filtered and evaporated at reduced pressure to yield residues of $23.5 \%$ based on the dry plant material. The extract was stored at $-18^{\circ} \mathrm{C}$ for experimental procedure. 


\section{Gas Chromatography-Mass Spectrometric Analysis}

The hydroalcoholic extract of MO was dissolved in absolute ethanol $(1 \mathrm{mg} / \mathrm{mL})$. A total of $10 \mu \mathrm{L}$ of this sample was then injected for gas chromatography-mass spectrometric (GC-MS) analysis.

The extract was analyzed using a Younglin Acm 6000 GC with HP-5MS column $(30 \mathrm{~m} \times 0.25 \mathrm{~mm}$, film thickness $0.25 \mu \mathrm{m})$; carrier gas, He; split ratio, 1:25; and using a flame ionization detector. GC-MS was executed on a HP 6890 with a HP 5973 quadrupole detector, on a capillary column HP-5MS; carrier gas, $\mathrm{He}$; flow rate, $0.8 \mathrm{~mL} / \mathrm{min}$; and column temperature, $50-240^{\circ} \mathrm{C}\left(3^{\circ} \mathrm{C}\right.$ $\left.\mathrm{min}^{-1}\right)$. The MS was performed at $70 \mathrm{eV}$ ionization energy. Retention indices were calculated using retention times of $n$-alkanes. The phytoconstituents of MO extract were identified based on the NIST and Wiley mass spectral library. 24,25

\section{Animals}

Male Wistar-albino rats, weighing 200-220 g were obtained from animal house of Faculty of Pharmacy at Tehran University of Medical Sciences (Tehran, Iran). Animals were housed in cages consisting of 6-7 rats per cage and were maintained under standard conditions of temperature $\left(23 \pm 1^{\circ} \mathrm{C}\right)$, relative humidity $(55 \pm$ $10 \%$ ), and 12/12-hour light/dark cycle, and allowed free access to a standard pellet chow and tap water. The experiments were performed in accordance with national guidelines for the use and care of laboratory animals and approved by the Animal Care Committee of Tehran University of Medical Sciences by No. 93-03-86-27155321902 .

\section{Induction of Experimental Models of Irritable Bowel Syndrome}

\section{Acetic acid experimental model}

A total number of 63 animals were assigned randomly to 9 groups with 7 rats in each group according to the following schemas.

In the first step of this study, induction of IBS was performed by instillation of acetic acid to induce IBS as described previously. ${ }^{26,27}$ Rats were left to recover from colitis for 6 days. On the 7 th day, the animals were assigned randomly to 8 groups to receive mentioned treatment for 3 consecutive days including (1) sham group which are normal animals, (2) the control group (acetic acidinduced IBS rats with no treatment) that received the same volume of saline instead of extract of MO intragastrically by gavage, (3) MO-100 group that received MO extract at dose of $100 \mathrm{mg} / \mathrm{kg}$ per day by gavage, (4) MO-200 group that received MO extract at dose of $200 \mathrm{mg} / \mathrm{kg}$ per day by gavage, (5) MO-300 group that received MO extract at dose of $300 \mathrm{mg} / \mathrm{kg}$ per day by gavage, (6) AG control group that received AG $(100 \mathrm{mg} / \mathrm{kg})$ intraperitoneally, (7) L-NAME control group that received the L-NAME (10 mg/ $\mathrm{kg}$ ) intraperitoneally, (8) MO-300 + AG group that received AG $(100 \mathrm{mg} / \mathrm{kg})$ intraperitoneally 1 hour before administration of MO300 via gastric tube once daily, and (9) MO-300 + L-NAME group that received L-NAME $(10 \mathrm{mg} / \mathrm{kg})$ intraperitoneally at 1 hour before administration of MO-300 via gastric tube once daily (Fig. 1). On day 11, evaluation of VH to rectal distension was carried out in each group by determination of abdominal withdrawal reflex (AWR) scores and also defecation patterns. At the end of procedures, histological samples were collected to determine the extent of colonic inflammation.

\section{Restraint stress model}

In order to induce IBS by the restraint stress model, animals were lightly anesthetized with inhalation of ether and the restraint was performed using plastic rodent restrainers that allowed for a close fit to rats in their home cages. Stress consisted of 6 hours of immobilization from $9 \mathrm{AM}$ to $3 \mathrm{PM}$ for 5 consecutive days in all groups except the sham group. As shown in Figure 1, a total number of 49 animals were randomly assigned to 7 groups with 7 rats in each group including (1) sham group which are normal animals; (2) the control group (restraint stress-induced IBS rats with no treatment) that received the same volume of normal saline instead of extract of MO intragastrically by gavage; (3) MO-300 group, as the most effective dose based on pilot study, that received MO extract at dose of $300 \mathrm{mg} / \mathrm{kg}$ per day by gavage; (4) AG control group that received AG (100 mg/kg) intraperitoneally; (5) LNAME control group, that received the L-NAME $(10 \mathrm{mg} / \mathrm{kg})$ intraperitoneally; (6) MO-300 + AG group that received AG (100 $\mathrm{mg} / \mathrm{kg}$ ) intraperitoneally 1 hour before administration of MO300 via gastric tube once daily; and (7) MO-300 + L-NAME group that received L-NAME (10 mg/kg) intraperitoneally 1 hour before administration of MO-300 through gastric tube once daily. On the 5 th day, after overnight fasting, animals were killed using an overdose of ether inhalation. The colons were removed, cut longitudinally and homogenized for biochemical and immunological investigations as previously described. ${ }^{28}$ Then, $100 \mu \mathrm{L}$ of the homogenates were taken for ferric reducing antioxidant power (FRAP) assay and frozen at $-80^{\circ} \mathrm{C}$ until analysis. The rest of the samples were sonicated and centrifuged for 30 minutes at $3500 \mathrm{~g}$. Then, the aliquoted supernatants were transferred into several microtubes and 


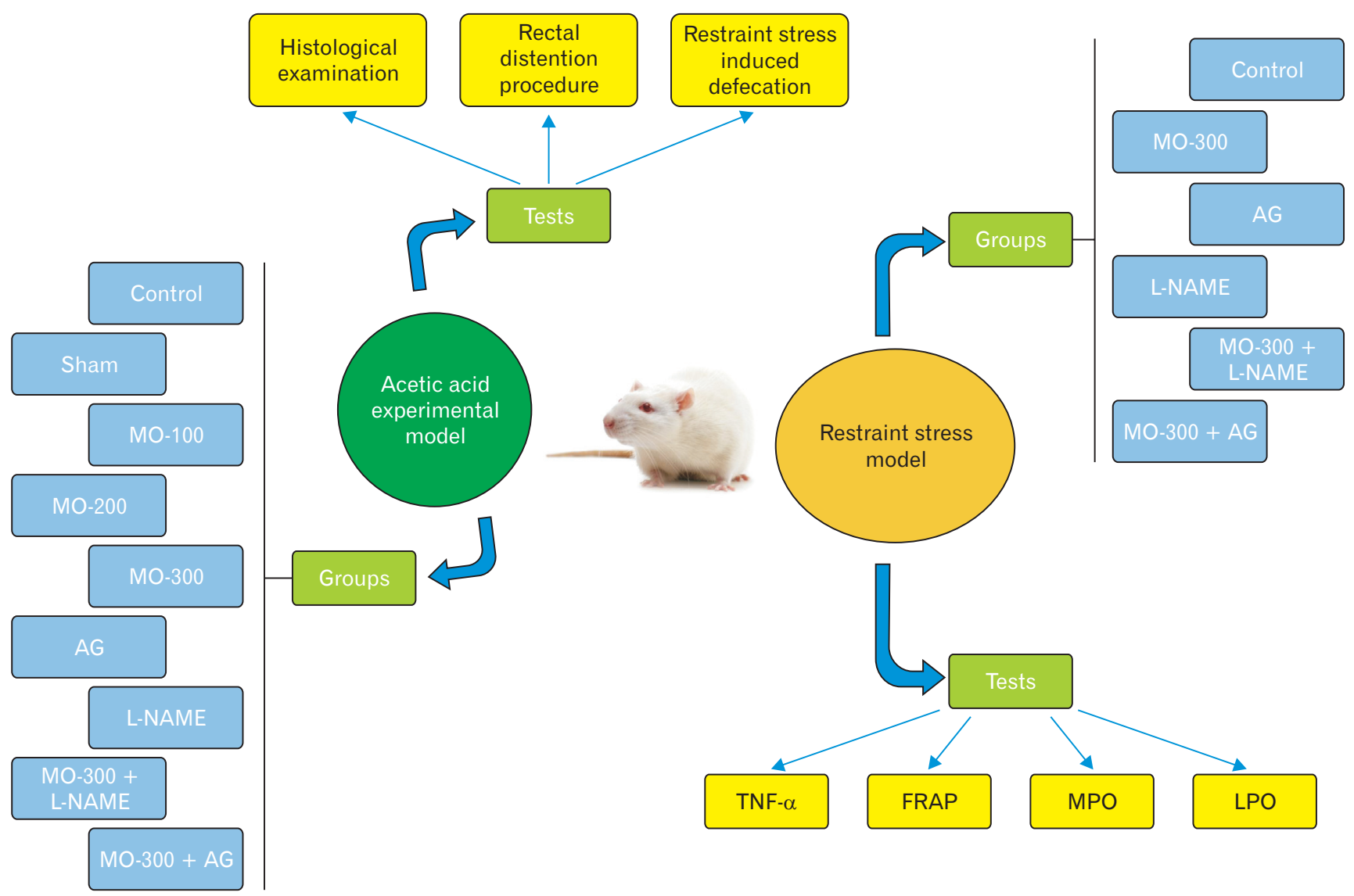

Figure 1. Diagram of study design. MO-100, group that received Melissa officinalis L. (MO) extract at dose of $100 \mathrm{mg} / \mathrm{kg}$ per day by gavage; MO-200, group that received MO extract at dose of $200 \mathrm{mg} / \mathrm{kg}$ per day by gavage; MO-300, group that received MO extract at dose of $300 \mathrm{mg} /$ kg per day by gavage; AG, aminoguanidine; L-NAME, L-NG-nitroarginine methyl ester; FRAP, ferric reducing antioxidant power; MPO, myeloperoxidase; LPO, lipid peroxides.

stored at $-80^{\circ} \mathrm{C}$ until analysesd. ${ }^{28,29}$

\section{Histological Examination of Inflammation}

To examine the extent of colonic inflammation, a colon segment of $1 \mathrm{~cm}$ in length was removed from the distal colon at the selected time points ( 2 days and 7 days post-enema in 2 rats of each group). ${ }^{27,30}$ Histological samples were fixed in $10 \%$ formalin. The coded histology slides were analyzed by a gastrointestinal pathologist.

\section{Rectal Distension Procedure}

On day 11, rats from each group were used for evaluating visceral sensitivity secondary to rectal distension as previously described. Then the AWR was semi-quantitatively scored according to previous experiments. ${ }^{26,27,31}$ The AWR score was assigned as follows: $0=$ no behavioral response to distension, $1=$ brief head movements followed by immobility, $2=$ contraction of abdominal muscle without lifting of abdomen, 3 = lifting of abdomen, and 4 = body arching and lifting of pelvic structure. The nociceptive threshold was defined as the minimal distension volume in which the rats were given an AWR score of 2.30

\section{Restraint Stress Procedure}

During the 1 hour of procedure of restraint stress that was performed on day 11 , rats were placed in restraint cages $(5 \mathrm{~cm} \times 5 \mathrm{~cm}$ $\times 20 \mathrm{~cm}$ ) while body movement of the rats were limited, but they had no problems with respiration. Then, the feces excreted were counted and stool consistency was evaluated in one of 3 categories: hard pellet, soft pellet, and formless stools. ${ }^{32}$ 


\section{Biochemical Assay}

\section{Tumor necrosis factor- $\alpha$ assay}

The amount of TNF- $\alpha$ was measured in the colon via ratspecific ELISA kit as described previously. ${ }^{33}$ Data were expressed as $\mathrm{pg} / \mathrm{mg}$ protein of tissue.

\section{Ferric reducing antioxidant power assay}

The total antioxidant capacity of the colon tissue homogenate was determined by measuring its ability to reduce $\mathrm{Fe}^{3+}$ to $\mathrm{Fe}^{2+}$ by the FRAP test as described in our previous work. ${ }^{34,35}$ Data were expressed as $\mathrm{mM}$ ferric ions reduced to ferrous per $\mathrm{mg}$ of protein.

\section{Myeloperoxidase activity assay}

MPO activity assay as a liposomal peroxidase enzyme that is released from neutrophil was done to determine the inflammation in colon tissue according to the procedure described previously. MPO activity was reported as $\mathrm{U} / \mathrm{mg}$ protein of tissue. ${ }^{34,35}$

\section{Thiobarbituric acid reactive substances (lipid perox- ides) assay}

Thiobarbituric acid reactive substances (TBARS) assay was used for measuring lipid peroxidation according to previously described method. $^{36}$
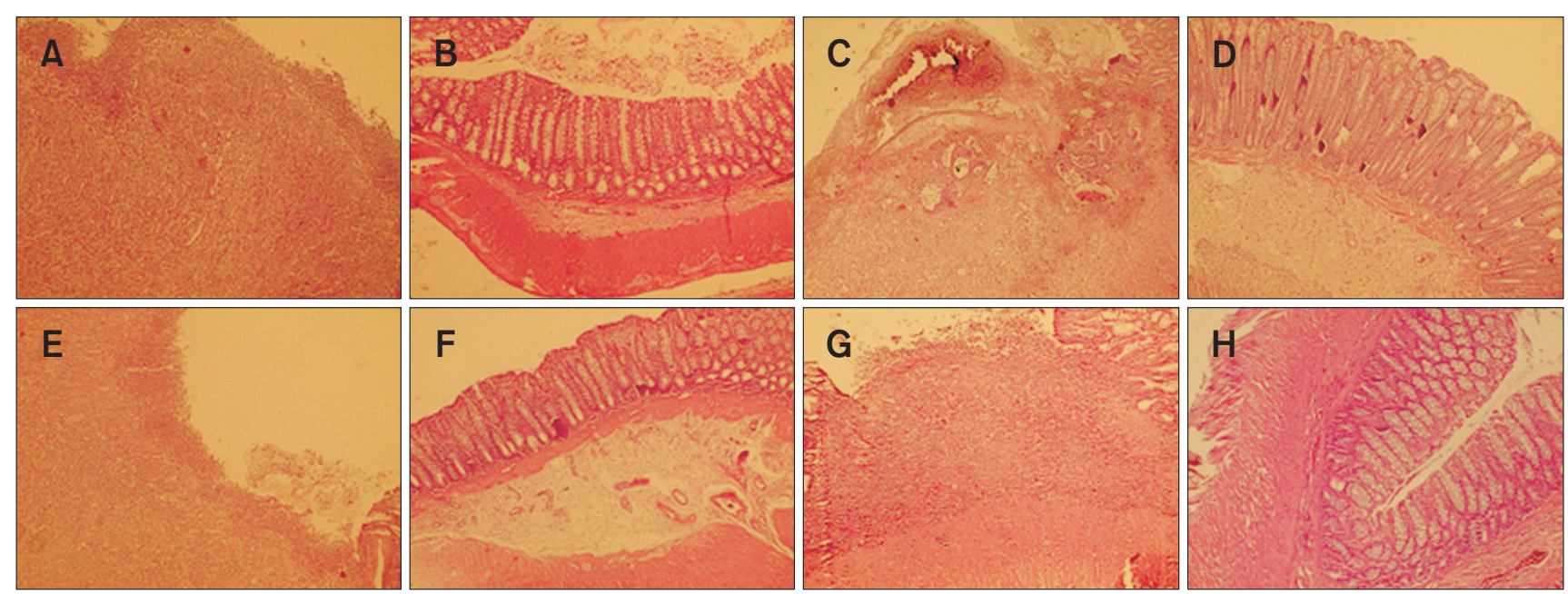

Figure 2. Distal colons at 2 (A) and 7 days (respectively, from aminoguanidine [AG] + Melissa officinalis L. [MO] groups [B], AG groups [C, D], L-NG-nitroarginine methyl ester [L-NAME] + MO groups [E, F], and from the L-NAME groups [G, H]) in acetic acid model. Original magnification, $\times 100$. 
and citronellal (3.43\%).

\section{Histological Examination of Inflammation}

Figure 2 shows the histology of the distal colon at days 2 and 7 after acetic acid instillation in all experimental groups. Histology of the distal colon 2 days after acetic acid instillation indicated complete degeneration of mucus, mucosal hemorrhage, damage of mucous glands, and surface of epithelium, including goblet cells, submucosal edema, and inflammatory infiltration in the lamina propria and the submucosa. At day 7 after induction of colitis, a large part of the structure of mucosa including mucous glands, surface of epithelium and goblet cells were regenerated and no signs of inflammation had observed. No significant inflammatory features were found 7 days after acetic acid instillation in each group.

\section{Assessment of Visceral Hypersensitivity}

The nociceptive threshold was $0.8 \mathrm{~mL}$ in the sham group and the MO-300 group, but it decreased to $0.4 \mathrm{~mL}$ in the control, MO-100, MO-200, AG, L-NAME, and AG + MO groups. The AWR score in the control group was significantly higher than the sham and MO-300 when distension volume was $0.4 \mathrm{~mL}$ and $0.6 \mathrm{~mL}(P<0.05)$. AWR score in MO-100 and MO-200, AG, and L-NAME + MO groups did not show significant decrease than the control group $(P<0.05)$ (Table 1$)$.

\section{Restraint Stress-induced Defecation}

Rats in the control group defecated a total of $8.50 \pm 1.93$ feces, while rats in the sham group defecated a total of $1.28 \pm 0.75$ feces. Therefore, rats in the control group had significantly increased defecation $(P>0.05)$ (Table 2$)$. A total of feces output in the AG group $(9.43 \pm 1.62)$ and L-NAME group $(8.71 \pm 1.50)$ showed significant increase in comparison to the sham group $(P>0.05)$ (Table 2).

Number of hard pellets, soft pellets, and total pellets in the MO-300 group showed that the stool form shifted from soft to hard, and decreased defecation frequency in comparison to the control group, but the MO-100 and MO-200 did not show significant change in frequency and form of stool when compared with the control group (Table 2). AG and L-NAME did not exhibit any considerable alteration in stool consistency patterns and stool frequencies $(P>0.05)$.

MO-300 + AG group showed significant decrease in defecation in comparison to the MO-300 and could effectively decrease the protective effect of $\mathrm{MO}$ on defecation (Table 2).

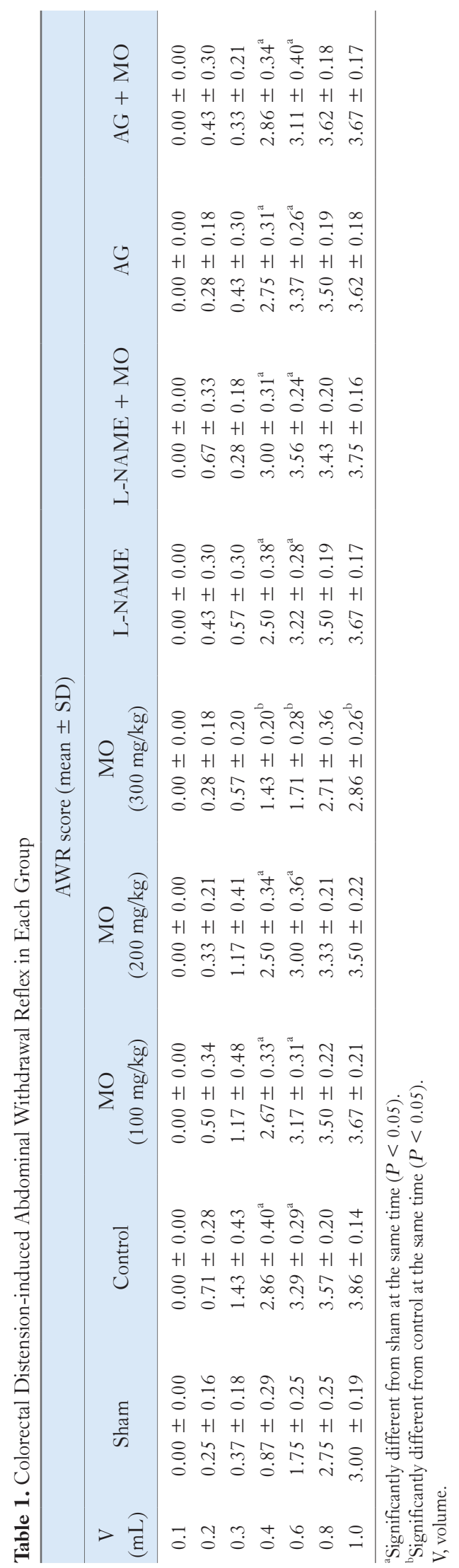



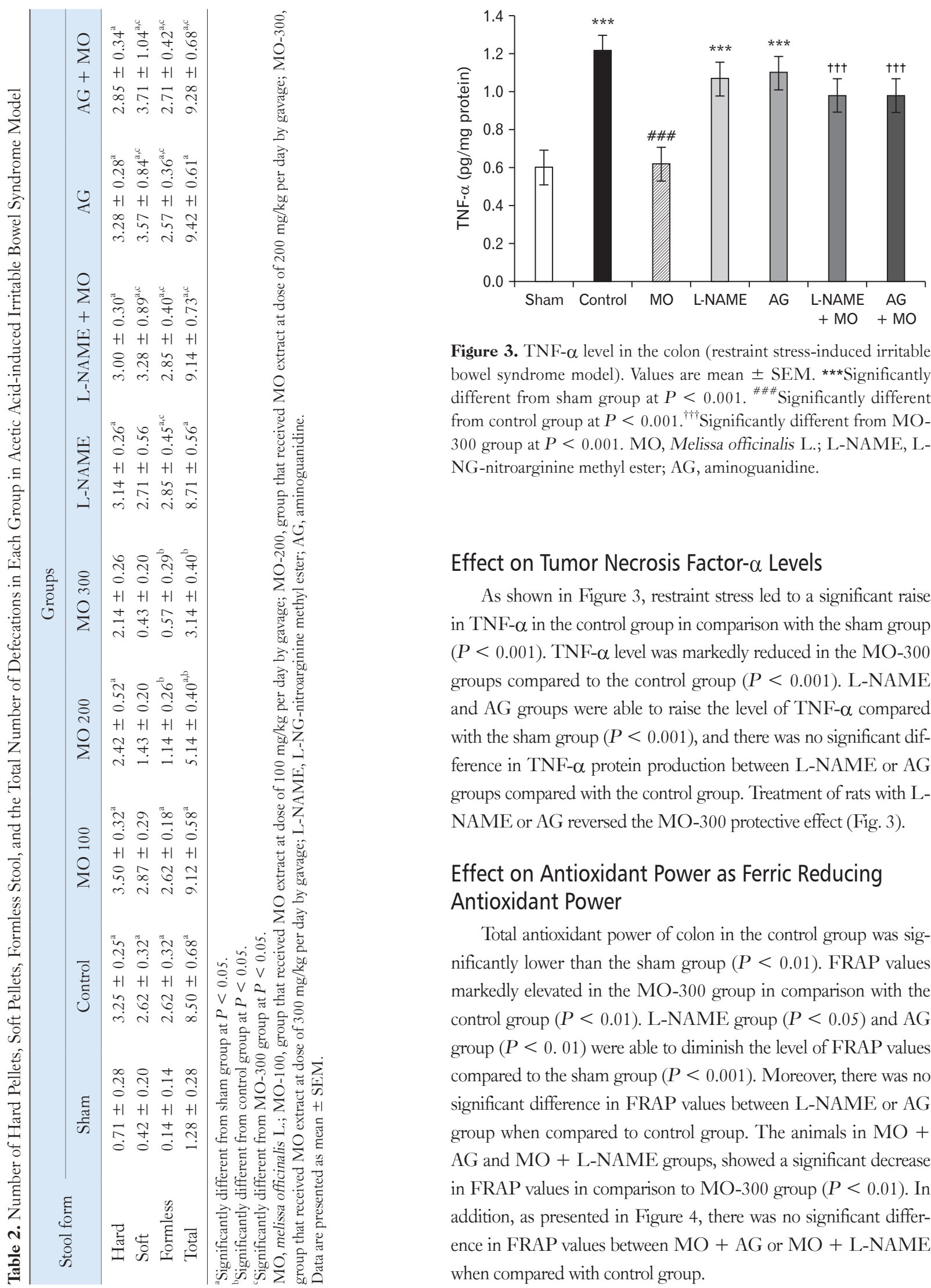

Figure 3. TNF- $\alpha$ level in the colon (restraint stress-induced irritable bowel syndrome model). Values are mean \pm SEM. *** Significantly

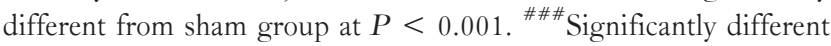
from control group at $P<0.001$. $^{\dagger \dagger}$ Significantly different from MO300 group at $P<0.001$. MO, Melissa officinalis L.; L-NAME, LNG-nitroarginine methyl ester; AG, aminoguanidine.

\section{Effect on Tumor Necrosis Factor- $\alpha$ Levels}

As shown in Figure 3, restraint stress led to a significant raise in TNF- $\alpha$ in the control group in comparison with the sham group $(P<0.001)$. TNF- $\alpha$ level was markedly reduced in the MO-300 groups compared to the control group $(P<0.001)$. L-NAME and AG groups were able to raise the level of TNF- $\alpha$ compared with the sham group $(P<0.001)$, and there was no significant difference in TNF- $\alpha$ protein production between L-NAME or AG groups compared with the control group. Treatment of rats with LNAME or AG reversed the MO-300 protective effect (Fig. 3).

\section{Effect on Antioxidant Power as Ferric Reducing Antioxidant Power}

Total antioxidant power of colon in the control group was significantly lower than the sham group $(P<0.01)$. FRAP values markedly elevated in the MO-300 group in comparison with the control group $(P<0.01)$. L-NAME group $(P<0.05)$ and AG group $(P<0.01)$ were able to diminish the level of FRAP values compared to the sham group $(P<0.001)$. Moreover, there was no significant difference in FRAP values between L-NAME or AG group when compared to control group. The animals in $\mathrm{MO}+$ AG and MO + L-NAME groups, showed a significant decrease in FRAP values in comparison to MO-300 group $(P<0.01)$. In addition, as presented in Figure 4, there was no significant difference in FRAP values between $\mathrm{MO}+\mathrm{AG}$ or $\mathrm{MO}+\mathrm{L}-\mathrm{NAME}$ when compared with control group. 


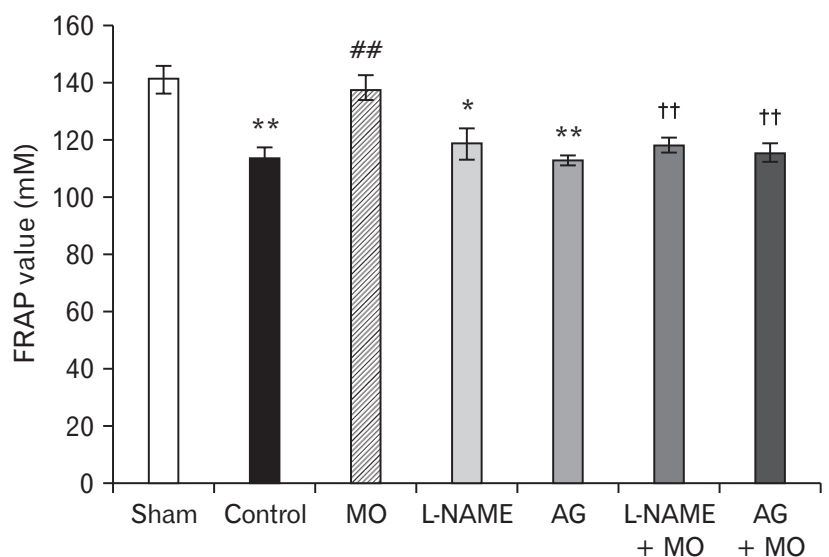

Figure 4. Ferric reducing antioxidant power (FRAP) level in the colon (restraint stress-induced irritable bowel syndrome model). Values are mean \pm SEM. *Significantly different from sham group at $P<0.05$. ${ }^{*}$ Significantly different from sham group at $P<0.01$.

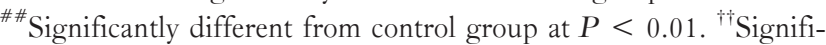
cantly different from group that received Melissa officinalis L. (MO) extract at dose $300 \mathrm{mg} / \mathrm{kg}$ per day by gavage (MO-300) at $P<0.01$. L-NAME, L-NG-nitroarginine methyl ester; AG, aminoguanidine.

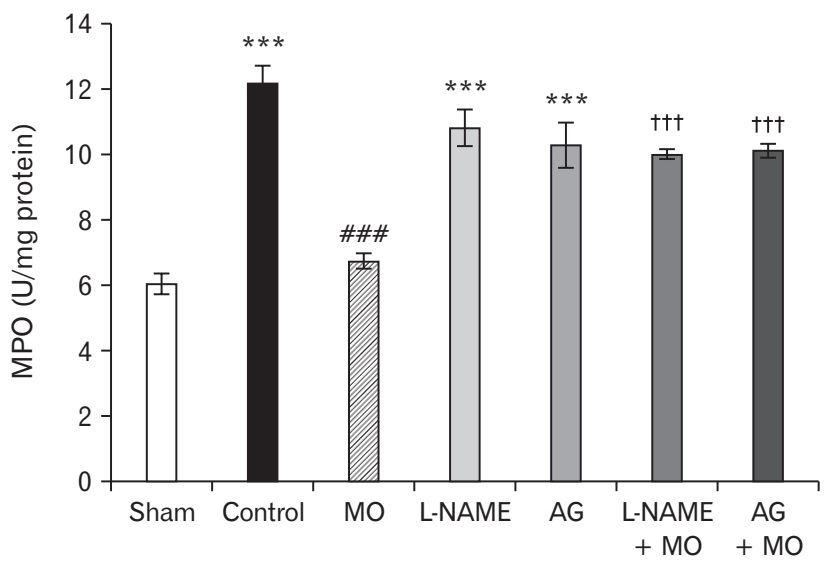

Figure 5. Myeloperoxidase (MPO) activity in the colon (restraint stress-induced irritable bowel syndrome model). Values are mean


\#\#\# Significantly different from control group at $P<0.001$. "Nignificantly different from group that received Melissa officinalis L. (MO) extract at dose $300 \mathrm{mg} / \mathrm{kg}$ per day by gavage (MO-300) at $P<0.001$. L-NAME, L-NG-nitroarginine methyl ester; AG, aminoguanidine.

\section{Effect on Myeloperoxidase Activity}

As depicted in Figure 5, colonic damage by restraint stress procedure was also indicated by an elevation in the MPO activity in the control group ( $P<0.001$, compared to sham group). $\mathrm{MPO}$ activity was significantly lower in the extract-treated group (300 mg/

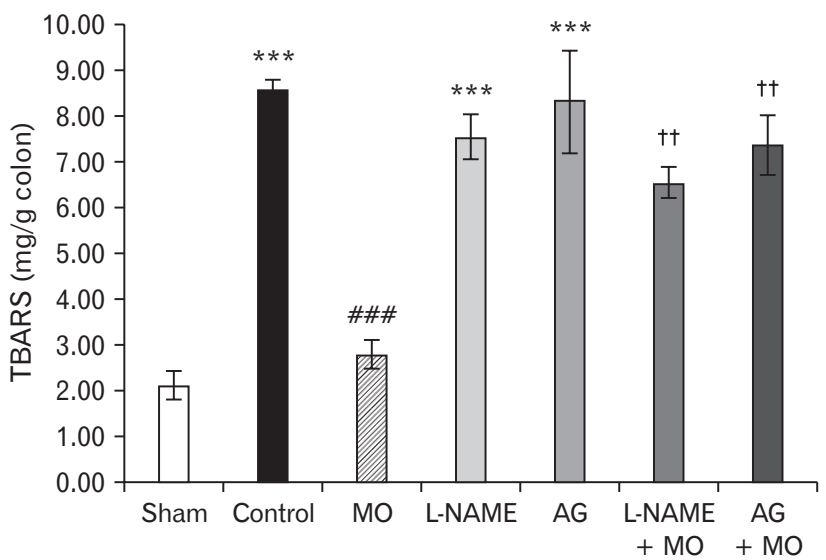

Figure 6. Thiobarbituric acid reactive substances (TBARS) level in the colon (restraint stress-induced irritable bowel syndrome model). Values are mean \pm SEM. ***Significantly different from sham group at $P<0.001$. \#\#\# Significantly different from control group at $P<$ 0.001. ${ }^{\dagger}$ Significantly different from group that received Melissa officinalis $\mathrm{L}$. (MO) extract at dose $300 \mathrm{mg} / \mathrm{kg}$ per day by gavage (MO$300)$ at $P<0.01{ }^{\dagger+1}$ Significantly different from MO-300 group at $P$ $<0.001$. L-NAME, L-NG-nitroarginine methyl ester; AG, aminoguanidine.

$\mathrm{kg})(P<0.001)$. MPO activity increased in the L-NAME and AG groups, which was significantly higher than the sham group $(P<0.001)$. There was no difference between the L-NAME and $\mathrm{AG}$ group and control groups. The animals in the $\mathrm{MO}+\mathrm{AG}$ and $\mathrm{MO}+\mathrm{L-NAME}$ groups showed a significant increase in $\mathrm{MPO}$ activity in comparison with the MO-300 group $(P<0.001)$ (Fig. 5).

\section{Effect on Oxidative-stress as Thiobarbituric Acid Reactive Substances}

TBARS was significantly higher in the control group compared with the sham group $(P<0.001)$. Its level was markedly decreased in the MO-300 group compared with the control group $(P$ $<0.001)$. In the other groups, the marker increased in a significant manner and TBARS levels in the L-NAME or AG group was similar to the control group. The animals in $\mathrm{MO}+\mathrm{AG}$ and $\mathrm{MO}$ + L-NAME groups, exhibited a significant increase in the level of TBARS compared to the MO-300 group $(P<0.001$ and $P<$ 0.01 , respectively). As presented in Figure 6, there was no significant difference between $\mathrm{MO}+\mathrm{AG}$ and $\mathrm{MO}+\mathrm{L}-\mathrm{NAME}$ when compared with the control group. 


\section{Discussion}

This study was designed to elucidate the effects of MO treatment on IBS signs (VH and defecation) in a post-inflammatory reaction with acetic acid and restraint stress model. Evaluation of bowel inflammatory reaction and oxidative stress biomarkers were performed so as to predict the potential therapeutic mechanisms of $\mathrm{MO}$ in IBS. The role of nitrergic pathway on the protective effects of MO using NOS inhibitors, aminoguanidine and L-NAME was also examined. According to modern investigations, a wide range of medicinal plants encompass positive activity in the management of gastrointestinal disorders as IBS ${ }^{38}$ Herbal medicines have demonstrated their effectiveness in managing IBS via various pharmacological mechanisms including modulating the immune system and proinflammatory cytokines (such as toll-like receptor, mast cells, and prostaglandins), $\mathrm{NO}$, and inducible isoform of NOS, nuclear factor-kappa B, opioid receptor, histamine receptor, adrenergic pathway, and gut microbiota, as well as anti-oxidative stress and antimicrobial activities. ${ }^{8}$

The model of diarrhea-predominant IBS conducted in the current experiment is on the basis of post-inflammatory induction of colonic dysmotility and $\mathrm{VH}$ in animals. In the evaluation of postinflammatory reaction with the acetic acid model, we employed colorectal distention stimulator and the distention stress processes as the pace from lower to higher and the AWR scoring system to objectively evaluate VH. In the control group, 7 days after instillation of acetic acid when no sign of inflammation was detected in the colon, rats showed $\mathrm{VH}$ and a high frequency of defecation (in response to restraint stress) with more soft and formless stool patterns compared to the sham group. These findings are in line with the clinical symptoms in IBS patients; however, there is a remarkable dilemma in utilizing this model to examine the effect of drugs on IBS ${ }^{26,30}$ Potential therapeutic function of natural agents on different signs of IBS including VH or defecation has mostly focused on these signs in colitis rather than IBS. To avoid this ambiguity, histopathological parameters of inflammation in each group, 7 days after acetic acid instillation, were measured and the complete subsidence of inflammation was observed in each experimental group. Considering the fact that administration of MO-300 significantly improved symptoms of IBS in rats, it could be concluded that MO had neither a positive nor negative activity on the histopathological factors of inflammation in the colon, and did not damage induction of the post-inflammatory model.

Restraint stress model was conducted based on the fact that stress and psychological tensions are involved in the pathogenesis of IBS, and have been performed to evaluate the effects of MO on bowel inflammatory reaction and oxidative stress pathological cascades. IBS is actually a stress-related disorder and stress is one of the most important factors in its pathogenesis. ${ }^{39}$

The hypothalamus-pituitary axis is the main stress axis in mammals which are located both in the CNS and in the periphery. In response to psychosocial or interoceptive stress, corticotropin releasing hormone (CRH) is released from the paraventricular nucleus of the hypothalamus and binds to CRH receptors (CRHR1 and CRHR2) in pituitary gland where it causes release of adrenocorticotropic hormone (ACTH) into systemic circulation. ACTH produces and secretes glucocorticoids from the adrenal cortex. Moreover, CRH affects stimulation of sympathetic nervous system and parasympathetic of the sacral nerve. Taken together, the results of induction of $\mathrm{CRH}$ release include elevated visceral sensitization, rectal hypersensitivity, as well as colonic motility. ${ }^{40}$ La et a ${ }^{41}$ reported that the post-inflammatory $\mathrm{VH}$ is mediated by the elevated peripheral CRH.

Restraint stress is one of the most generally employed model for the induction of both acute and chronic stress in rats. This is a modified form of immobilization stress in which animals are not permitted to move for a definite period of time. Stress in a model of immobilization is a complex of physical and psychological stressor. ${ }^{42}$ We have used a well-known model of stress-induced IBS model in rats to evaluate the effect of $\mathrm{MO}$ on the level of TNF- $\alpha$, MPO, and TBARS, as well as the total antioxidant power of colonic tissues. ${ }^{28} \mathrm{TNF}-\alpha$ levels in the treatment group diminished significantly following $\mathrm{MO}$ treatment. In restraint stress associated IBS, the expression of pro-inflammatory cytokines are significantly increased. It has been confirmed that the pro-inflammatory cytokines affect the function of sensory innervations as well as enteric nerves with the stimulation of irregular secretomotor responses within the bowel, resulting in altered pain threshold and visceral sensory perception that can lead to the generation of gut pain and discomfort. ${ }^{6,43}$

Production of reactive oxygen species (ROS) and free radicals is recognized to occur in inflammation and lipid peroxidation. ${ }^{44}$ It has been found that ROS as an irritant and inflammatory mediator is able to lead to a state of $\mathrm{VH}^{45}$ In evaluating the role of oxidative stress in the pathogenesis of IBS, Mete et $\mathrm{al}^{46}$ reported that IBS impaired the oxidant-antioxidant enzymatic system. Another study in patients with post-infectious-IBS revealed that enhancing the levels of antioxidants can reduce IBS signs with decrease of ROS. ${ }^{45,47}$ Our results have demonstrated that $\mathrm{MO}$ can remarkably diminish the 
level of lipid peroxidation, in terms of TBARS, and increase level of tissue antioxidant performance (FRAP), indicating the role of oxidative stress associated factors in the therapeutic effect of $\mathrm{MO}$. MO has shown effective antioxidant and radical scavenging activities and these properties have been attributed to flavonoids and phenolic compounds. $^{48,49}$

Studies have demonstrated that animals' intestinal sensorymotor system is changed after induction of experimental colitis and these alterations persist even after relief of inflammation. ${ }^{50}$ Also, a role has been assumed for low-grade inflammation in both the pathogenesis and generation of symptoms associated with IBS. ${ }^{51}$ Acetic acid-induced colitis model was selected as it mimics numerous pathophysiological abnormalities that have been defined as characteristics of post-inflammatory IBS such as VH, motility dysfunction and change in permeability or secretion. ${ }^{50}$

Various studies reported that MO could be useful in the management of various inflammatory disease and pain. ${ }^{17,52}$ Although the exact mechanism(s) used in these studies are unclear, inhibition of the L-arginine-nitric oxide pathway and activation of cholinergic (eg, nicotinic and muscarinic acetylcholine receptors) systems seems to have an important role in its antinociceptive effect. ${ }^{12}$

The outcomes of the present study indicate the role of the NO pathway in the protective effects of MO using 2 NOS inhibitors (amino guanidine and L-NAME). Our results revealed that NOS inhibitors significantly block the positive effects of $\mathrm{MO}$ on $\mathrm{VH}$, oxidative stress and inflammatory markers, indicating that $\mathrm{MO}$ exerts its beneficial effects in IBS through NO-dependent mechanism.

NO has a pivotal role in modulation of nociception and maintaining $\mathrm{VH}$ in animal and human studies. ${ }^{53}$ Dai et $\mathrm{al}^{26}$ indicated that administration of the NOS inhibitor, AG, lowers the protective effect of VSL\# 3 as a probiotic on VH. In another similar study, a NOS inhibitor, L-NAME, inhibited the beneficial effects of pioglitazone on $\mathrm{VH}$ and defecation of rats. ${ }^{32} \mathrm{NO}$ also plays a key role in regulating gastrointestinal motility, which is essential for stool formation. ${ }^{26,53}$ Some studies have suggested that the nitrergic pathway is associated with mucosal inflammation. NO donating agents stimulate electrolyte secretion in the bowel tissue, which is partly mediated by expression of prostaglandin E2. Exogenous NO can diminish expression of inflammatory cytokines as well as neutrophil adherence and infiltration. ${ }^{6,7,32}$

The use of only male rats and the failure to measure various cytokines involved in the pathogenesis of IBS were to limitations of this study. So, it is suggested to design other experimental studies involved both male and female rats and investigate the effects of MO on other cytokines including IL-1 $\beta$, IL-6, IL-8, and IL-12 and other inflammatory pathways rather than nitrergic one.

In conclusion, the present study has demonstrated that $\mathrm{MO}$ effectively reduced $\mathrm{VH}$ to colorectal distension, diminished pain thresholds, reduced defecation frequency, and altered stool consistency toward hard pellets. MO effectively reduced the level of inflammatory parameters and lipid peroxidation along with enhanced mucosal antioxidant capability to confront the oxidative stress. Investigating the role of $\mathrm{NO}$ in the protective effects of $\mathrm{MO}$ using NOS inhibitors showed that NOS inhibitors noticeably reduced the effect of MO on clinical signs of IBS, inflammatory cytokines, and also antioxidant capability which indicates the remarkable role of the nitrergic pathway in therapeutic function of MO. Results obtained from the current study suggest that MO extract may be helpful in treating IBS in humans. Clinical trials are required to confirm this efficacy.

Financial support: This study has been partially supported by Tehran University of Medical Sciences (TUMS) (Grant No. 9303-86-27155).

\section{Conflicts of interest: None.}

Author contributions: Roja Rahimi and Amir H Abdolghaffari designed the study; Fatemeh Dolatabadi, Mohammad H Farzaei, Maryam Baeeri, Fatemeh S Ziarani, and Majid Eslami conducted the study; Mohammad H Farzaei and Maryam Baeeri analyzed data; Fatemeh Dolatabadi and Amir H Abdolghaffari drafted the manuscript; and Roja Rahimi and Mohammad Abdollahi revised the draft and approve the final version of the manuscript.

\section{References}

1. Endo Y, Shoji T, Fukudo S. Epidemiology of irritable bowel syndrome. Ann gastroenterol 2015;28:158-159.

2. Bokic T, Storr M, Schicho R. Potential causes and present pharmacotherapy of irritable bowel syndrome: an overview. Pharmacology 2015;96:7685.

3. O'Malley D. Immunomodulation of enteric neural function in irritable bowel syndrome. World J Gastroenterol 2015;21:7362-7366.

4. Liebregts T, Adam B, Bredack C, et al. Immune activation in patients with irritable bowel syndrome. Gastroenterology 2007;132:913-920.

5. Kim JH, Lin E, Pimentel M. Biomarkers of irritable bowel syndrome. J Neurogastroenterol Motil 2017;23:20-26.

6. Farzaei MH, Bahramsoltani R, Abdollahi M, Rahimi R. The role of visceral hypersensitivity in irritable bowel syndrome: pharmacological targets and novel treatments. J neurogastroenterol motil 2016;22:558-574 . 
7. Li S, Fei G, Fang X, et al. Changes in enteric neurons of small intestine in a rat model of irritable bowel syndrome with diarrhea. J Neurogastroenterol Motil 2016;22:310-320.

8. Kuiken SD, Klooker TK, Tytgat GN, Lei A, Boeckxstaens GE. Possible role of nitric oxide in visceral hypersensitivity in patients with irritable bowel syndrome. Neurogastroenterol Motil 2006;18:115-122.

9. Rahimi R, Abdollahi M. Herbal medicines for the management of irritable bowel syndrome: a comprehensive review. World J Gastroenterol 2012;18:589-600.

10. Bahramsoltani R, Farzaei MH, Farahani MS, Rahimi R. Phytochemical constituents as future antidepressants: a comprehensive review. Rev Neurosci 2015;26:699-719.

11. R, Farzaei MH, Rahimi R. Medicinal plants and their natural components as future drugs for the treatment of burn wounds: an integrative review. Arch Dermatol Res 2014;306:601-617.

12. Guginski G, Luiz AP, Silva MD, et al. Mechanisms involved in the antinociception caused by ethanolic extract obtained from the leaves of Melissa officinalis (lemon balm) in mice. Pharmacol Biochem Behav 2009;93:10-16.

13. Shakeri A, Sahebkar A, Javadi B. Melissa officinalis L.- a review of its traditional uses, phytochemistry and pharmacology. J Ethnopharmacol 2016;188:204-228.

14. Farzaei MH, Rahimi R, Abdolladi Z, Abbasabahi Z. An evidence-based review on medicinal plants used for the treatment of peptic ulcer in traditional iranian medicine. Int J Pharmacol 2013;9:108-124.

15. Sadraei H, Ghannadi A, Malekshahi K. Relaxant effect of essential oil of Melissa officinalis and citral on rat ileum contractions. Fitoterapia 2003;74:445-452.

16. Drozd J, Anuszewska E. The effect of the Melissa officinalis extract on immune response in mice. Acta Pol Pharm 2003;60:467-470.

17. Bounihi A, Hajjaj G, Alnamer R, Cherrah Y, Zellou A. In vivo potential anti-inflammatory activity of melissa officinalis L. essential oil. Adv Pharmacol Sci 2013;2013:101759.

18. Hossain MA, Kim S, Kim KH, Lee SJ, Lee H. Flavonoid compounds are enriched in Lemon balm (Melissa officinalis) leaves by a high level of sucrose and confer increased antioxidant activity. Hortscience 2009;44:1907-1913.

19. Ibarra A, Feuillere N, Roller M, Lesburgere E, Beracochea D. Effects of chronic administration of Melissa officinalis L. extract on anxiety-like reactivity and on circadian and exploratory activities in mice. Phytomedicine 2010;17:397-403.

20. Sarris J, Panossian A, Schweitzer I, Stough C, Scholey A. Herbal medicine for depression, anxiety and insomnia: a review of psychopharmacology and clinical evidence. Eur Neuropsychopharmacol 2011;21:841-860.

21. Taiwo AE, Leite FB, Lucena GM, et al. Anxiolytic and antidepressantlike effects of Melissa officinalis (lemon balm) extract in rats: influence of administration and gender. Indian J Pharmacol 2012;44:189-192.

22. Dastmalchi K, Dorman HD, Oinonen PP, Darwis Y, Laakso I, Hiltunen $\mathrm{R}$. Chemical composition and in vitro antioxidative activity of a lemon balm (Melissa officinalis L.) extract. LWT Food Sci Technol 2008;41:391-400.

23. Farzaei MH, Khazaei M, Abbasabadei Z, Feyzmahdavi M, Mohseni
GR. Protective effect of tragopogon graminifolius DC against ethanol induced gastric ulcer. Iran Red Crescent Med J 2013;15:813-816.

24. Farzaei MH, Rahimi R, Attar F, et al. Chemical composition, antioxidant and antimicrobial activity of essential oil and extracts of tragopogon graminifolius, a medicinal herb from Iran. Nat prod commun 2014;9:121-124.

25. Basu S, Das M, Sen A, Choudhury UR, Datta G. Analysis of complete nutritional profile and identification of bioactive components present in Alocasia indica tuber cultivated in howrah district of west bengal, India. Asian Pac J Trop Med 2014;7:S527-S533.

26. Dai C, Guandalini S, Zhao DH, Jiang M. Antinociceptive effect of VSL\#3 on visceral hypersensitivity in a rat model of irritable bowel syndrome: a possible action through nitric oxide pathway and enhance barrier function. Mol Cell Biochem 2012;362:43-53.

27. La JH, Kim TW, Sung TS, Kang JW, Kim HJ, Yang IS. Visceral hypersensitivity and altered colonic motility after subsidence of inflammation in a rat model of colitis. World J Gastroenterol 2003;9:2791-2795.

28. Larauche M, Mulak A, TachéY. Stress-related alterations of visceral sensation: animal models for irritable bowel syndrome study. J Neurogastroenterol Motil 2011;17:213-234.

29. Ponferrada A, Caso JR, Alou L, et al. The role of PPARgamma on restoration of colonic homeostasis after experimental stress-induced inflammation and dysfunction. Gastroenterology 2007;132:1791-1803.

30. Shamshiri H, Paragomi P, Paydar MJ, et al. Antinociceptive effect of chronic lithium on visceral hypersensitivity in a rat model of diarrheapredominant irritable bowel syndrome: the role of nitric oxide pathway. J Gastroenterol Hepatol 2009;24:672-680.

31. Al-Chaer ED, Kawasaki M, Pasricha PJ. A new model of chronic visceral hypersensitivity in adult rats induced by colon irritation during postnatal development. Gastroenterology 2000;119:1276-12785.

32. Paragomi P, Rahimian R, Kazemi MH, et al. Antinociceptive and antidiarrheal effects of pioglitazone in a rat model of diarrhoea-predominant irritable bowel syndrome: role of nitric oxide. Clin Exp Pharmacol Physiol 2014;41:118-126.

33. Farzaei MH, Ghasemi-Niri SF, Abdolghafari AH, et al. Biochemical and histopathological evidence on the beneficial effects of tragopogon graminifolius in TNBS-induced colitis. Pharm Biol 2015;53:429-436.

34. Nakhai LA, Mohammadirad A, Yasa N, et al. Benefits of zataria multiflora boiss in experimental model of mouse inflammatory bowel disease. EBCAM 2007;4:43-50.

35. Hasani P, Yasa N, Vosough-Ghanbari S, Mohammadirad A, Dehghan G, Abdollahi M. In vivo antioxidant potential of Teucrium polium, as compared to $\alpha$-tocopherol. Acta pharm 2007;57:123-129.

36. Astaneie F, Afshari M, Mojtahedi A, et al. Total antioxidant capacity and levels of epidermal growth factor and nitric oxide in blood and saliva of insulin-dependent diabetic patients. Arch Med Res 2005;36:376-381.

37. Bradford MM. A rapid and sensitive method for the quantitation of microgram quantities of protein utilizing the principle of protein-dye binding. Anal Biochem 1976;72:248-254.

38. Sodagari HR, Farzaei MH, Bahramsoltani R, Abdolghaffari AH, Mahmoudi M, Rezaei N. Dietary anthocyanins as a complementary medicinal approach for management of inflammatory bowel disease. Expert 
Rev Gastroenterol Hepatol 2015;9:807-820.

39. Moloney RD, O'Mahony SM, Dinan TG, Cryan JF. Stress-induced visceral pain: toward animal models of irritable-bowel syndrome and associated comorbidities. Front Psychiatry 2015;6:15.

40. Fukudo S. Role of corticotropin-releasing hormone in irritable bowel syndrome and intestinal inflammation. J Gastroenterol 2007;42(suppl 17):48-51.

41. La JH, Sung TS, Kim HJ, Kim TW, Kang TM, Yang IS. Peripheral corticotropin releasing hormone mediates post-inflammatory visceral hypersensitivity in rats. World J Gastroenterol 2008;14:731-736.

42. Bali A, Jaggi AS. Preclinical experimental stress studies: protocols, assessment and comparison. Eur J Pharmacol 2015;746:282-292.

43. Sarris J, McIntyre E, Camfield DA. Plant-based medicines for anxiety disorders, part 2: a review of clinical studies with supporting preclinical evidence. CNS drugs 2013;27:301-319.

44. Oran M, Tulubas F, Mete R, Aydin M, Sarikaya HG, Gurel A. Evaluation of paraoxonase and arylesterase activities in patients with irritable bowel syndrome. J Pak Med Assoc 2014;64:820-822.

45. Han W, Lu X, Jia X, Zhou T, Guo C. Soluble mediators released from PI-IBS patients' colon induced alteration of mast cell: involvement of reactive oxygen species. Dig Dis Sci 2012;57:311-319.

46. Mete R, Tulubas F, Oran M, et al. The role of oxidants and reactive nitrogen species in irritable bowel syndrome: a potential etiological explana- tion. Med sci Monit 2013;19:762-766.

47. Berstad A, Raa J, Valeur J. Tryptophan: 'essential' for the pathogenesis of irritable bowel syndrome? Scand J Gastroenterol 2014;49:1493-1498.

48. Hossain MA, Kim S, Kim KH, Lee S-J, Lee H. Flavonoid compounds are enriched in lemon balm (Melissa officinalis) leaves by a high level of sucrose and confer increased antioxidant activity. Hortscience 2009;44:1907-1913.

49. Pereira RP, Fachinetto R, de Souza Prestes A, Puntel RL, da Silva GNS, Heinzmann BM, et al. Antioxidant effects of different extracts from Melissa officinalis, Matricaria recutita and Cymbopogon citratus. Neurochem Res 2009;34:973-983.

50. Qin HY, Wu JC, Tong XD, Sung JJ, Xu HX, Bian ZX. Systematic review of animal models of post-infectious/post-inflammatory irritable bowel syndrome. J Gastroenterol 2011;46:164-174.

51. Ford AC, Talley NJ. Mucosal inflammation as a potential etiological factor in irritable bowel syndrome: a systematic review. J Gastroenterol 2011;46:421-431.

52. Birdane YO, Buyukokuroglu ME, Birdane FM, Cemek M, Yavuz H. Anti-inflammatory and antinociceptive effects of Melissa officinalis L. in rodents. Rev Med Vet 2007;158:75-81.

53. Kuiken SD, Klooker TK, Tytgat GN, Lei A, Boeckxstaens GE. Possible role of nitric oxide in visceral hypersensitivity in patients with irritable bowel syndrome. Neurogastroenterol Motil 2006;18:115-122. 